\title{
The motor homunculus: linking the past with the present
}

\author{
Michaela Wilson, Haejin Dadachanji, Davin Greenwell
}

Since the 1870 s the primary motor cortex has been investigated for its functional architecture and how it relates to motor movement. Though the motor cortex had been observed to contain a sort of organized somatotopy in earlier studies, the relationship between cortical organization and motor control was still unclear. Advancements in stimulation techniques propelled the investigations and discoveries of specific cortical maps that represented different areas of the body. The most well-known (and generally accepted) of these discoveries were by Penfield and Rasmussen who, through invasive stimulation of the primary motor cortex, proposed a somatotopic relationship between areas of the motor cortex and areas of the body that they controlled. Penfield and Rassmusen's motor homunculus details a medial to lateral band of the motor cortex broken down into specific segments representing individual body parts including the separation of the fingers (thumb, index, middle, ring and pinky finger) and demonstrates distinct boundaries between somatotopic representations of different body parts. Importantly, Penfield and Rasmussen's observations were made in awake operated subjects, something that is rare, particularly in intact humans without possible pre-existing damage (e.g. epilepsy). Though Penfield and Rasmussen's work was groundbreaking at the time, the field of neuroscience has come a long way since the 1950s and several limitations have become apparent in light of more modern techniques.

More recent ( $<50$ years) understanding of motor cortical organization comes from activation studies where either the brain is stimulated non-invasively, or the subject is asked to perform (or imagine) a

This is the author's manuscript of the work published in final edited form as:

Wilson, M., Dadachanji, H., \& Greenwell, D. (2021). The motor homunculus: Linking the past with the present. The Journal of Physiology, 599(6), 1731-1732. https://doi.org/10.1113/JP280982 
certain task while the brain is imaged. Each of these, while meritorious, has its specific limitations when examining the exact organization of the primary motor cortex. For instance, a commonly used noninvasive stimulation technique is transcranial magnetic stimulation. Without careful placement of the stimulation coil, guided by previous mapping techniques, stimulation is relatively inexact compared to microstimulation of the surface of the brain invasively. Furthermore, the relative intensities required to activate specific regions are likely to cause more widespread activation of surrounding brain tissues. Imaging techniques present their own problems, as now the accuracy of the response in the brain region is limited to how finite the movement is that is being performed (or imagined). Although these techniques are incredibly useful for understanding the general structure and function of the brain, in this context they simply do not provide the resolution needed to confirm, refute or further expand on Penfield and Rasmussen's initial findings.

Since the time of Penfield and Rasmussen's work, we have a much better understanding of the connections and circuitry of the motor cortex. For example, we now know that there is not a 1:1 or direct processing relationship between the motor cortex and muscles (Schieber, 2001). This can be observed with principles such as convergence and divergence. Convergence refers to the joint activation of specific motor action by two or more separate cortical motor sites. Inversely, divergence refers to the synchronous activation of differing motor outputs by stimulation of a single cortical site. The presence of these properties of the motor cortex, coupled with the evidence for an individual's cortical plasticity and reorganization presents a case for a sort of relativity in individual somatotopy.

Another aspect to consider with regard to somatotopic organization is the presence of negative motor areas. Stimulation of negative motor areas results in the inhibition of, instead of the activation of, 
movement. Rech et al. (2019) proposed the presence of a large-scale modulatory function primarily located in the motor cortex. In that study, they found central areas in which negative motor movements were evoked. These sites were located rostrally to positive motor areas with significant overlap and appeared to descend deep into the white matter of the brain.

Considering the limitations posed by Penfield and Rasmussen's work in motor-compromised individuals, and considering the significant advancements in technology, medicine and neuroscience in the past 60 years, the motor homunculus has been long overdue for a reassessment. No large-scale systematic mapping studies of the human primary motor cortex have been attempted in awake human subjects recently. This is likely due, in part, to the extraordinary challenge and time commitment that such a task presents. In a recent study in The Journal of Physiology, Roux et al. (2020) collected data that spanned the course of 14 years. Rigorous inclusion and exclusion standards were required to ensure that subjects had no significant motor deficits despite lesions in other areas of the brain, thereby allowing this dataset to reflect a standard motor homunculus. There would be significant challenges to reproducing a similar study and it is for this very reason that Roux et al.'s work is so important in understanding the human motor somatotopy.

Roux et al. sought to 'update' the functional areas of the motor homunculus and its relationship with the somatosensory homunculus using coordinates in the standard Montreal Neurological Institute (MNI) space. They tested 100 patients without motor deficits or brain lesions on the precentral gyrus that were surgically undergoing brain tumour removal elsewhere. Using direct electrical stimulation of the motor cortex in these awake subjects, they relied on patient feedback (patients indicated feeling something) rather than recorded electromyograms (EMGs) directly from the muscle. The patient 
feedback and observation was similar to the methods Penfield and Rasmussen used for linking cortical sites to motor actions, but may not have detected weaker responses that could have been observed in EMG recordings (Yingling et al. 1999)

Of the 608 sites stimulated on the precentral gyrus, 248 (40\%) were positive points for motor movement (Roux et al. 2020). There are many factors that could explain why most of the sites were unresponsive to stimulation: small undetectable responses (explained in the previous paragraph), activation of negative motor areas, and insufficient current intensity (detailed later). In line with previous work, most of the positive sites were in small clusters on the cortex and had little variability between subjects. Specifically, these small clusters for most body parts were centred in relation to the top of the precentral gyrus and highly localized. The movements observed were found to be stereotypical, isolated and basic. For example, flexion was produced more often than extension, specifically in the digits and wrist.

Similar to the work of Penfield and Rasmussen, Roux et al. confirmed that the face, lips and tongue areas took up a large region of the motor homunculus. However, Penfield and Rasmussen noted a large region associated with movement of the lips, tongue and jaw that additionally resulted in the involuntary production of vocal noise (described as loud and sudden sound) (Penfield \& Rasmussen, 1950). This was not confirmed in the present study, though the current intensities used in this study were much lower, likely resulting in fewer 'false-positive' responses due to less current spread to neighbouring areas. This issue of stimulation intensity is a double-edged sword, however, because there may have been insufficient current to cause motor movements that could have been otherwise observed with EMGs. 
In contrast to previous research, Roux et al. showed consistent somatotopic organization of movement within the precentral gyrus, and specifically demonstrated a relatively medial to lateral somatotopy of hand motor function. Penfield and Rasmussen, on the other hand, suggested a degree of variability in the somatotopic representation of the precentral gyrus; however, they failed to provide a clear explanation for what was defined as 'variability'. Roux et al. defined variability as either 'aberrant somatotopic organization' or inconsistency in the, 'localization of... cortical representation(s) of movement within the precentral gyrus'. By these definitions, they assert that little inter-individual variability was observed in their findings and that most of the variability that was observed could be explained by somatotopy.

Another interesting point was that Roux et al. demonstrated that only $2 \%$ of the motor responses observed were from stimulating outside of the precentral gyrus, whereas Penfield and Rasmussen reported much higher values ( 20\%). Again, this difference is likely best explained with the higher stimulation intensities used by Penfield and Rasmussen that led to greater current spread to surrounding areas. Alternatively, the current study reported that responses to stimulation could be entirely intensity-dependent, as they found motor movements evoked with an intensity of $2.2 \pm 0.4 \mathrm{~mA}$ were then inhibited with an intensity of $2.26 \pm 0.5 \mathrm{~mA}$. These negative motor areas (Rech et al. 2019) were never considered in earlier experiments, but have to be examined now based on the findings of Roux et al. Such a small change in electrical current can potentially activate totally contradictory motor functions. This is certainly important enough to be considered in future motor mapping studies.

The work by Roux and colleagues is the first in a long time to provide the scientific community with an updated somatotopic model of the human motor homunculus. Their work bridges the 'old' and the 
'new' in terms of the groundwork that people like John Hughlings Jackson, Sir Charles Sherrington and Wilder Penfield have done. Considering the meticulous subject selection, data collection and simply overall time that Roux and colleagues put into this study, it is an important piece in advancing our understanding of the organization of the brain relative to the control of movement. 


\section{References}

Penfield W \& Rasmussen T (1950). The Cerebral Cortex of Man: A Clinical Study of Localization of Function. MacMillan Publishing Co., New York.

Rech F, Herbet G, Gaudeau Y, Mezieres S, Moureau J-M, Moritz-Gasser S \& Duffau H (2019). A probabilistic map of negative motor areas of the upper limb and face: a brain stimulation study. Brain 142, 952- 965.

Roux F-E, Niare M, Charni S, Giussani C \& Durand J-B (2020). Functional architecture of the motor homunculus detected by electrostimulation. J Physiol 598, 5487- 5504.

Schieber MH (2001). Constraints on somatotopic organization in the primary motor cortex. J Neurophysiol 86, 2125- 2143.

Yingling CD, Ojemann S, Dodson B, Harrington MJ \& Berger MS (1999). Identification of motor pathways during tumor surgery facilitated by multichannel electromyographic recording. $J$ Neurosurg 91, 922-927. 